\title{
Editorial
}

Z Gerontol Geriat 2013 · 46:4-4 DOI 10.1007/s00391-012-0456-6

Online publiziert: 16. Dezember 2012

(c) Springer-Verlag 2012

A. Motel-Klingebiel · J.P. Ziegelmann

Deutsches Zentrum für Altersfragen, Berlin

\section{Die Lebensphase Hochaltrigkeit}

se an Erkenntnissen beitragen kann. Im zweiten Beitrag „Die soziale Konstruktion von ,Hochaltrigkeit' in einer jungen Altersgesellschaft" geht Amrhein der Frage nach, inwieweit Hochaltrigkeit in Ergänzung zu biologischen und psychologischen Perspektiven auch anhand soziologischer Kriterien definiert werden kann, und illustriert dies mit empirischen Beispielen. Fürstenberg konstatiert in seinem Artikel „Hochaltrigkeit als Akzeptanzproblem" eine Gefahr der gesellschaftlichen Ausgrenzung Hochbetagter und zeigt Wege zur Förderung sozialer Akzeptanz und Inklusion auf. Beide Arbeiten tragen zur Weiterentwicklung von konzeptionellen Zugängen bei. Im vierten Beitrag stellen Bennett $u$. Riedel die Lebenssituationen Hochbetagter in den Mittelpunkt und betrachten sie vom Standpunkt des Verhältnisses von Versorgung und Lebenszufriedenheit. Sie geben auf der Grundlage umfassender Daten aus der deutschsprachigen Schweiz einen empirischen Einblick in die ambulante Altenpflege und -hilfe und diskutieren Möglichkeiten einer optimalen Abstimmung formeller und informeller Betreuung.

Das vorliegende Heft soll mit seinen Themenbeiträgen sowohl wichtige Arbeiten zum hohen Alter vorstellen als auch Anregungen dazu geben, Forschung als interdisziplinäres Projekt anzulegen und die Analyse der Pfade in die sehr späten Lebensphasen und zu Lebenssituationen Hochbetagter in Deutschland zu intensivieren.

Nicht zuletzt möchten wir den Herausgebern und der Redaktion der Zeitschrift für Gerontologie und Geriatrie danken, dass wir diese theoretischen und empirischen Zugänge zur Hochaltrigkeit der interdisziplinären Leserschaft präsentieren können. Hierbei danken wir vor al- lem auch Clemens Tesch-Römer für seine sehr konstruktiven Hinweise zu den ersten Fassungen der Themenbeiträge.

Sicher können die Beiträge nur Schlaglichter auf das Themenfeld der Hochaltrigkeit werfen. Dennoch hoffen wir, dass die vorliegenden Arbeiten dazu anregen, die Pfade in die Hochaltrigkeit sowie Lebenssituationen Hochbetagter in ihren individuellen wie gesellschaftlichen Dynamiken künftig auf einer breiten theoretisch-konzeptionellen Grundlage besser $\mathrm{zu}$ verstehen und zu ihrer individuellen wie gesellschaftlichen Gestaltung beizutragen.
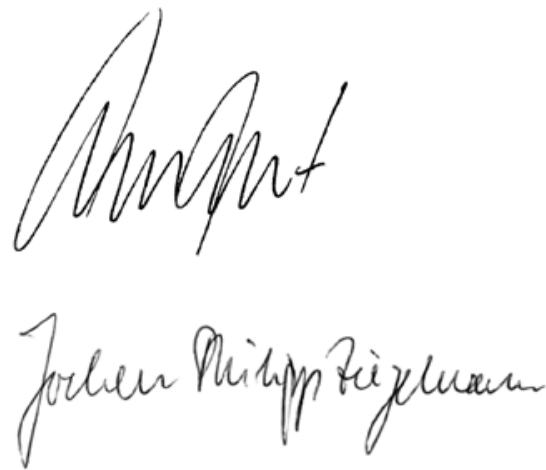

\section{Korrespondenzadressen}

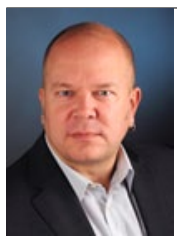

PD Dr. A. Motel-Klingebiel

Deutsches Zentrum

für Altersfragen

Manfred-von-Richthofen-Str. 2, 12101 Berlin andreas.motel-klingebiel@ dza.de

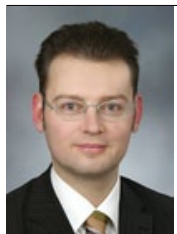

Dr. J.P. Ziegelmann Deutsches Zentrum für Altersfragen Manfred-von-Richthofen-Str. 2, 12101 Berlin jochen.ziegelmann@dza.de 